\title{
A new method of transient stability assessment in power systems using LS-SVM
}

\begin{abstract}
This paper presents transient stability assessment of electrical power system using least squares support vector machine (LS-SVM) and principle component analysis. Transient stability of a power system is first determined based on the generator relative rotor angles obtained from time domain simulation outputs. Simulations were carried out on the IEEE 9bus test system considering three phase faults on the system. The data collected from the time domain simulations are then used as inputs to the LS-SVM in which LS-SVM is used as a classifier to determine the stability state of a power system. Principle component analysis is applied to extract useful input features to the LS-SVM so that training time of the LS-SVM can be reduced. To verify the effectiveness of the proposed LS-SVM method, its performance is compared with the multi layer perceptron neural network. Results show that the LS-SVM gives faster and more accurate transient stability assessment compared to the multi layer perceptron neural network in terms of classification results.
\end{abstract}

Keyword: Least squares support vector machines; Artificial neural network; Dynamic security assessment; Time domain simulation method; Transient stability assessment 